\title{
Anmerkungen zum Wahlrechtsstreit - Ein Problem gelöst, ein anderes bleibt. Oder: Ein Blick über die Grenzen lehrt Gelassenheit
}

\section{Hubert Kleinert}

Seit dem Wahlrechtsurteil des Bundesverfassungsgerichts aus dem Sommer 2008 wird in Deutschland über die Neufassung des Wahlrechts zur Bundestagswahl gestritten. ${ }^{1}$ Darüber ist die von Karlsruhe dem Gesetzgeber gesetzte Frist zur Wahlrechtsänderung verstrichen. Erst im September 2011 haben schließlich Union und FDP einen Gesetzentwurf in den Bundestag eingebracht, der auf die Ablehnung der Oppositionsparteien gestoßen ist. Während die Regierungsfraktionen nur die Verrechnung von Zweistimmenanteilen zwischen den einzelnen Bundesländern korrigieren und feste Mandatsanteile für die 16 Länder einführen wollen, die sich nach der Wahlbeteiligung richten, sollen nach Willen der SPD im Fall von Überhangmandaten künftig Ausgleichsmandate vergeben und damit die bei vielen Landtagswahlen geübte Praxis auch auf den Bund übertragen werden. Die Grünen wollen Überhangmandate generell beseitigen und dies im Wege einer Verrechnung von Überhangmit Listenmandaten in anderen Bundesländern erreichen. Wo dies nicht möglich (CSU) oder nicht ausreichend ist (wenn zu viele Überhangmandate entstehen), will man jenen mit der Erststimme eigentlich gewählten Wahlkreisabgeordneten das Mandat nicht zuteilen, die mit dem geringsten Stimmenanteil gewählt worden sind.

Trotz langwieriger Verhandlungen kam es nicht zu einer Verständigung zwischen den Parteien. So beschloss der Bundestag am 29. September 2011 den Entwurf der Regierungsmehrheit und der Bundesrat ließ die Wahlrechtsänderung passieren. Doch SPD und Grüne halten die Neuregelung nicht für verfassungsgemäß und haben erneut das Bundesverfassungsgericht angerufen. So ist bislang nicht gewiss, nach welchem Wahlrecht der nächste Bundestag gewählt werden wird. Im Blick auf die angesichts der anhaltenden Probleme in der Bundesregierung nicht ganz unwahrscheinliche Möglichkeit einer vorgezogenen Parlamentsaufösung ist das ein verfassungsrechtlich bedenklicher Umstand.

Im Vordergrund der öffentlichen Debatte standen und stehen seit 2008 vor allem zwei Aspekte: Zum einen wird auf die ganz realistische Problematik hingewiesen, dass die beob-

1 Stellvertretend für die Fülle seither erschienener Abhandlungen, Stellungnahmen und Diskussionen zum Wahlrecht seien hier nur die in dieser Zeitschrift veröffentlichten genannt: Dieter Nohlen, Erfolgswertgleichheit als fixe Idee oder: Zurück zu Weimar? Zum Urteil des Bundesverfassungsgerichts über das Bundeswahlgesetz vom 3. Juli 2008, in: ZParl, 40. Jg. (2009), H. 1, S. 179 - 195; Joachim Behnke, Überhangmandate und negatives Stimmgewicht: Zweimannwahlkreise und andere Vorschläge, in: ZParl, 41. Jg. (2010), H. 2, S. 247 - 260; Franz Urban Pappi $/$ Michael Herrmann, Überhangmandate ohne negatives Stimmgewicht: Machbarkeit, Wirkungen, Beurteilung, in: ZParl, 41. Jg. (2010), H. 2, S. 260 - 278; Daniel Lübbert, Negative Stimmgewichte bei der Bundestagswahl, in: ZParl, 41. Jg. (2010), H. 2, S. 278 - 289; Gerd Strohmeier, Die Geister, die Karlsruhe rief - eine Replik auf die Beiträge zur Wahlsystemreform in Heft 2/2010 der ZParl, in: ZParl, 42. Jg. (2011), H. 1, S. 186 - 193; Joachim Behnke / Florian Grotz, Das Wahlsystem zwischen normativer Begründung, empirischer Evidenz und politischen Interessen. Ein Kommentar zu Gerd Strohmeier sowie Franz Urban Pappi und Michael Herrmann, in: ZParl, 42. Jg. (2011), H. 2, S. 419 - 425; Daniel Lübbert / Felix Arndt / Friedrich Pukelsheim, Proporzwahrende Anpassung der Bundestagsgröße - ein Lösungsvorschlag für das Problem der negativen Stimmgewichte bei Bundestagswahlen, in: ZParl, 42. Jg. (2011), H. 2, S. 426 - 435. 
achtbare Fragmentierung des Parteiensystems die Verzerrungswirkungen der Überhangmandate verschärfen könnte und damit Überhangmandate zum wahlentscheidenden Faktor werden könnten. Zum anderen wird mit allerhand Rechenmodellen über die theoretisch mögliche Reichweite eines „negativen Stimmengewichts“ gestritten, das nur in besonderen und vergleichsweise leicht zu verhindernden Einzelfällen wirklich praktische Relevanz haben kann. Die grundlegenden Fragen nach den politischen Zwecken des personalisierten Verhältniswahlrechts und nach der politischen Bedeutung von Wahlrechten in der Demokratie und eines veränderten Parteiensystems geraten dabei nicht selten aus dem Blick.

\section{Das Urteil des BVerfG}

Das Urteil vom 3. Juli 2008 (BVerfGE 121, 266)² nahm Anstoß an $₫ 7$ Abs. 3, Satz 2 (alt) des Bundeswahlgesetzes, insoweit dieser in Verbindung mit $\$ 6$ Abs. 4 und 5 die Möglichkeit des negativen Stimmengewichts begründet. Den Anlass boten Beschwerden gegen die Gültigkeit der Wahl zum 16. Deutschen Bundestag. Diese Beschwerden machten sich vor allem daran fest, dass es durch die in einem Dresdener Wahlkreis erforderliche Nachwahl zwei Wochen nach dem eigentlichen Wahltermin tatsächlich zu einer besonderen Form der Verzerrung des Bundestagswahlergebnisses und der Mandatsverteilung gekommen war. Weil diese Nachwahl nämlich in Kenntnis des bundesweiten Gesamtergebnisses stattfand, konnte der Effekt des ansonsten nur theoretischen negativen Stimmengewichts - unter dem der Umstand verstanden wird, dass die Nichtwahl der eigentlich präferierten Partei mit der Zweitstimme ihr nutzt, während eine Wahl ihr schadet - bewusst eingesetzt werden.

Dass die Nichtwahl einer Partei mit der Zweitstimme dieser ein zusätzliches Mandat verschafft, während eine Stimmabgabe zu ihren Gunsten sie ein Mandat kosten kann, ist derart offenkundig widersinnig, dass es zwingend erscheint, diese Möglichkeit zu unterbinden. Insoweit sind die Überlegungen des Verfassungsgerichts treffend und nachvollziehbar.

Problematischer wird das Urteil allerdings insoweit, als es nicht allein auf Fälle wie Dresden abhebt, wo die Nutzung des negativen Stimmengewichts zugunsten einer Partei eine bewusst einsetzbare taktische Option war, sondern auch andere Konstellationen einbezieht, in denen aufgrund der Entstehung von Überhangmandaten die bloß theoretische Möglichkeit der Nutzung des negativen Stimmengewichts besteht. Einerseits verwirft das Verfassungsgericht keineswegs die Möglichkeit der Entstehung von Überhangmandaten und die damit immer verbundenen Verzerrungen im Erfolgswert jeder abgegebenen Wählerstimme; andererseits aber wird durch die Gleichsetzung der bewusst einsetzbaren Möglichkeit der taktischen Nutzung des negativen Stimmengewichts etwa durch einen Wahlaufruf zugunsten einer anderen Partei im Sonderfall einer Nachwahl mit rein theoretischen Möglichkeiten, die für Wahlmotive von Bürgern praktisch gar keine Rolle spielen können, diese Problematik durch die Hintertür wieder eingeführt. Will das Verfassungsgericht einerseits seiner Linie aus den 1990er Jahren treu bleiben, die die mit Überhangmandaten grundsätzlich verbundene Verletzung der Erfolgswertgleichheit jeder Stimme für hinnehmbar erklärt hat, so hat der Urteilstenor in Verbindung mit den aktuellen Entwicklungen des Parteiensystems die Tür für eine breite Grundsatzdebatte geöffnet, in deren Mittelpunkt die prinzipi-

2 Auch BVerfG 2 BvC 1/07 und 2 BvC 7/07 („Negatives Stimmgewicht“); vgl. auch Abdruck in: NJW 2008, S. 2700; DVBl 2008, S. 1045; NVwZ 2008, S. 991. 
elle Problematik von Überhangmandaten und ihr möglicher Effekt für die Bildung von Parlamentsmehrheiten gerückt ist.

Diese Vermengung erscheint jedoch keineswegs zwingend. Wer die Nutzung des negativen Stimmengewichts als bewusst einsetzbares taktisches Kalkül verhindern oder stark erschweren will, muss vor allem die Möglichkeit einer Nachwahl unterbinden - etwa durch die Verpflichtung der Parteien zur Nominierung von Ersatzbewerbern. Wenn aber das System der Ober- und Unterverteilung der Mandate als Ganzes moniert wird, wird ein Verfahren für verfassungswidrig erklärt, das jahrzehntelang unbeanstandet praktiziert worden ist. Dass das Verfassungsgericht bewusst einsetzbare taktische Kalküle mit dem Effekt von Verrechnungsverfahren gleichsetzt, die dem Wähler normalerweise weder bekannt sind noch von Wahlstrategen vorhergesehen werden können, erscheint insoweit keineswegs zwingend - zumal taktische Kalküle in den Entscheidungen der Wählerschaft seit der Einführung der Zweitstimme 1953 eine Rolle spielen. Theoretisch stieg und steigt die Chance der Union zur Erringung von Überhangmandaten mit jeder Erststimme, die der FDP-Anhänger dem CDU-Kandidaten, und mit jeder Zweitstimme die der CDU-Wähler der FDP gibt.

\section{Schieflagen in der öffentlichen Debatte}

Weil das Verfassungsgericht die Problematik der Überhangmandate mit dem tatsächlich nur in Sonderfällen praktisch relevanten Problem des negativen Stimmengewichts vermengt, ist die Debatte seither von einer Schieflage geprägt. Im Mittelpunkt steht weniger die Verhinderung derart absurder Ausnahmesituationen wie bei der Dresdener Nachwahl von 2005, sondern die Verletzung des Grundsatzes vom annähernd gleichen Erfolgswert jeder Stimme in einem System des personalisierten Verhältniswahlrechts, das eine besondere Form des Verhältniswahlrechts darstellt, deren Eigenart in der Aufnahme von Elementen des Mehrheitswahlrechts besteht. Dabei entsteht häufig der Eindruck, als könne der alleinige Sinn eines demokratischen Wahlrechts a priori nur darin bestehen, eine Mandatsverteilung zu ermöglichen, die die Kräfteverhältnisse in der Wählerschaft möglichst exakt widerspiegelt. Das Verfassungsgericht leistet dieser Richtung der öffentlichen Diskussion insoweit Vorschub, als es selbst betont, dass „alle Wähler mit den Stimmen, die sie abgeben, den gleichen Einfluss auf das Wahlergebnis haben“3 sollen. „Zur Zählwertgleichheit tritt im Verhältniswahlrecht die Erfolgswertgleichheit hinzu. ${ }^{\text {4 }}$

Tatsächlich aber besteht im Ziel, das Kräfteverhältnis in der Wählerschaft exakt im Parlament widerzuspiegeln, ein besonders wichtiger, aber keineswegs der einzige Zweck eines demokratischen Wahlrechts. Wer das wünscht, sollte das Wahlrecht der Weimarer Republik wieder einführen. Neben diesen Zweck treten Überlegungen, die mit politischer Handlungsfähigkeit, den Möglichkeiten zur einfachen und verlässlichen Mehrheitsbildung, dem Interesse an einer stabilen Regierungsarbeit sowie den Möglichkeiten einer dem föderalen System entsprechenden, regional ausgewogenen Rückbindung der Abgeordneten in die Wählerschaft zu tun haben. Diese verschiedenen und nie widerspruchsfrei zu verbindenden Ziele werden in unterschiedlichen demokratischen Wahlrechtsformen in unterschiedlichen Demokratien verschieden gewichtet. Mehrheitswahlrecht kennt keinen gleichen Erfolgs-

3 BVerfG 2 BvC 1/07 und 2 BvC 7/07, Rn. 92.

4 Ebenda, Rn. 93. 
wert jeder Stimme; alle Stimmen, die in Wahlkreisen für unterlegene Bewerber abgegeben werden, fallen unter den Tisch und sind für die Mandatsverteilung ohne Belang. Relevant ist hier allein die annähernd gleiche Größe der Wahlkreise. Auch Sperrklauseln in Systemen mit Verhältniswahlrecht haben den gleichen Effekt; Stimmen für Parteien, die an der Mandatsverteilung nicht teilnehmen, spielen keine Rolle, haben also den Erfolgswert null.

Zwar ist es richtig, dass in allen Systemen des Verhältniswahlrechts der Aspekt der proportionalen Repräsentation der gesellschaftlichen Grundströmungen in den Parlamenten im Vordergrund steht. Aber annähernd exakt umsetzen ließe sich das entweder nur in einem reinen Verhältniswahlrecht, das nur ein Wahlgebiet kennt, eine reine Listenwahl darstellt und auch keine Sperrklausel hat, oder in einem System großer, zumindest größerer Wahlkreise, in denen mehrere Abgeordnete gewählt werden. Solche Wahlsysteme existieren in Skandinavien. Ein reines Verhältniswahlrecht gab es annährend in der Weimarer Republik. Heute lässt es sich am ehesten in den Niederlanden finden.

Mit jedem anderen Wahlrecht aber sind gewisse Verzerrungen im Erfolgswert jeder Stimme verbunden. Dies gilt natürlich dann auch für Mischformen von Mehrheits- und Verhältniswahlrecht. Im Grabenwahlrecht, das das Verfassungsgericht als eine Möglichkeit zur Neugestaltung ausdrücklich erwähnt, wird dieser Grundsatz zum Beispiel schon insoweit verletzt, als nur die Hälfte der Mandate nach dem Zweitstimmenergebnis der Parteien verteilt würden.

Das personalisierte Verhältniswahlrecht, das die Ministerpräsidenten der Länder im Sommer 1949 gegen die Wünsche der westalliierten Militärgouverneure durchsetzten, wollte vier Ziele miteinander verbinden: Erstens die annähernd exakte Widerspiegelung der Grundstimmungen in der Wählerschaft im Parlament; zweitens die Verhinderung von Parteienzersplitterung und Stärkung der Handlungsfähigkeit von Parlament und daraus hervorgehenden Regierungen durch eine Sperrklausel; drittens eine einigermaßen proportionale Repräsentation der verschiedenen Regionen in der jeweils unterschiedlichen Stärke und Vielfalt der einzelnen Parteien und viertens die Rückbindung der einzelnen Abgeordneten an jeweils regional einigermaßen überschaubare Wahlkreise. Dem letzten Gesichtspunkt sollte durch die mit der Wahl von Wahlkreisabgeordneten verbundenen Elemente des Mehrheitswahlrechts entsprochen werden.

Weil dies die Intentionen des personalisierten Verhältniswahlrechts sind, müssen sich alle Reformvorschläge daran messen lassen, inwieweit sie diesen Rechnung tragen. Ein Grundtenor der Debatte, der so tut, als gehe es allein um das erste Ziel, geht daran vorbei.

\section{Zur gesetzlichen Neuregelung und den Alternativen der Opposition}

Die auf Initiative der Regierungsfraktionen verabschiedete Neuregelung will dem Urteil des Bundesverfassungsgerichts dadurch Rechnung tragen, dass sie die bisherige Praxis der Verrechnung des Mandatsanteils zwischen den einzelnen Landeslisten aufgibt und den Ländern einen je nach Wahlbeteiligung zu ermittelnden festen Mandatsanteil zuweist. Dieser ist entsprechend dem regionalen Stimmenergebnis auf die einzelnen Parteien zu verteilen. Im Falle von Überhangmandaten bliebe es bei der derzeitigen Praxis, dass diese als zusätzliche Mandate hinzukämen. Allerdings hätten sie keinen Einfluss auf die Mandatsverteilung in anderen Ländern. Als Sonderregelung ist ferner vorgesehen, dass eine Kumulierung von Nachkommastellen in den verschiedenen Ländern zusätzliche Mandate ergeben kann. 
Dieser Vorschlag trägt den Anforderungen des Verfassungsgerichts insoweit Rechnung, als er die Wirkungen des negativen Stimmengewichts auf rein theoretische Ausnahmefälle begrenzt. Er ändert allerdings nichts an den Verzerrungseffekten der Überhangmandate, will sich auf „minimal-invasive“ Eingriffe beschränken und die Chance zu einer angesichts der längerfristigen Entwicklungen des Parteiensystems überzeugenderen Gesamtlösung nicht ergreifen. Unverständlich erscheint zudem, dass man keine Vorkehrungen treffen will, um Nachwahlen wie in Dresden künftig zu verhindern oder mindestens zu erschweren.

Die Grünen haben bereits vor zwei Jahren einen Vorschlag vorgelegt, der inzwischen noch verschärft wurde. Er will die Entstehung von Überhangmandaten und damit alle Verzerrungseffekte künftig verhindern. Dies soll dadurch erreicht werden, dass in einzelnen Ländern entstehende Überhangmandate künftig mit von dieser Partei errungenen Listenmandaten in anderen Ländern verrechnet werden. Soweit dies nicht möglich ist - wie bei der CSU, die ja nur in Bayern kandidiert - oder nicht ausreicht - die Anwendung dieser Verrechnung hätte schon bei der Bundestagswahl 2009 dazu geführt, dass die CDU kein einziges Listenmandat errungen hätte -, sollen die direkt gewählten Abgeordneten der betroffenen Partei, die den relativ geringsten Stimmenanteil erzielt haben, dieses Mandat nicht bekommen.

Dieser Vorschlag löst ein gravierendes Problem, lässt aber zwei neue entstehen. Zum einen würde dieses Verrechnungsverfahren dazu führen, dass auch große Parteien unter Umständen in ganzen Regionen keine Abgeordneten mehr stellen würden. So hätte etwa die CDU im derzeitigen Bundestag keinen Bremer Abgeordneten mehr und im Flächenland Brandenburg nur einen einzigen. Der Gesichtspunkt der regional angemessenen Verteilung der Abgeordneten wäre bei Fraktionen mit einer größeren Zahl von Überhangmandaten stark gestört. Das hätte erhebliche Folgen. Zweitens ist es mindestens system-, wenn nicht verfassungswidrig, erfolgreichen Wahlkreisbewerbern das Mandat zu versagen: Zum System des personalisierten Verhältniswahlrechts gehört zwingend, dass jeder Wahlkreis einen eigenen direkt gewählten Abgeordneten stellt. Oder sollen die Wähler dafür bestraft werden, dass es in einem großstädtischen Wahlkreis womöglich ein politisch interessantes Kopf-anKopf-Rennen von drei oder gar vier chancenreichen Bewerbern gegeben hat? Kann es angehen, dass in einigen Wahlkreisen alle Erststimmen den Erfolgswert Null haben? Warum soll ein bestimmter Teil der Wählerschaft womöglich gar keinen Wahlkreisabgeordneten haben? $\mathrm{Zu}$ einer echten Wahl gehört, dass wenigstens einer gewählt wird, soweit dabei alles mit rechten Dingen zugeht. Die Idee der Grünen erscheint in diesem Punkt abwegig und ist gerade einer interessierten Wählerschaft kaum zu vermitteln.

Der Vorschlag der Sozialdemokraten zielt darauf ab, die Verzerrungseffekte von Überhangmandaten durch die Einführung von Ausgleichsmandaten zu korrigieren und damit die Regelungen, die in vielen Bundesländern existieren, auf den Bund zu übertragen. Auch dieser löst ein Problem, indem er zwei neue entstehen lässt. Überhangmandate führen zu einer Vergrößerung des Parlaments. Werden als Kompensation Ausgleichsmandate vergeben, wird das Parlament noch größer. Hinzu tritt eine gewaltige Verzerrung der regionalen Proportionen, die ihrerseits erhebliche Rückwirkungen auf den gleichen Erfolgswert der Stimmen hat, der doch gerade hergestellt werden soll. Wenn etwa die Verzerrungseffekte, die im derzeitigen Bundestag mit den vielen CDU-Überhangmandaten in Baden-Württemberg verbunden sind, dadurch geheilt werden sollen, dass die politischen Konkurrenten weitere Mandate zugeteilt bekämen, würde sich das unangemessene proportionale Gewicht Baden-Württembergs im Bundestag noch zusätzlich stark vergrößern. Ohne Überhangmandate stünden dem Bundesland heute 74 Mandate zu. Infolge der Überhangmandate ist 
diese Zahl in 2009 auf 84 gestiegen. Wollte man entsprechend dem SPD-Vorschlag Ausgleichsmandate vergeben, so säßen über 100 Abgeordnete aus Baden-Württemberg im Bundestag. Damit läge der Erfolgswert jeder dort abgegebenen Stimme bei allen Parteien, die in die Mandatsverteilung kommen, um circa 25 Prozent über dem Erfolgswert von Wählerstimmen in Ländern ohne Überhangmandate.

Selbst wer das Argument der zusätzlichen Parlamentsaufblähung nicht als entscheidend ansieht, kann nicht wollen, dass am Ende vier oder fünf Bundesländer mit vielen Überhangmandaten die Hälfte des Bundestags stellen.

Sicher sind Union und FDP mit ihrem Vorschlag sehr kurz gesprungen. Sie wollen so wenig wie möglich am geltenden Wahlrecht verändern. Dabei dürfte die Tatsache, dass unter den derzeit absehbaren Rahmenbedingungen des politischen Systems die Union die größte Chance hat, von Überhangmandaten zu profitieren, eine Rolle spielen. Das mag unbefriedigend sein, zumal es die Zukunftsprobleme nicht angeht. Die Gegenvorschläge der Opposition aber sind erst recht untauglich, weil sie die vorhandenen Probleme lösen wollen, aber neue aufwerfen, die gravierender sind als die, die doch gerade beseitigt werden sollen.

\section{Entscheiden die Überhangmandate die Regierungsbildung von morgen?}

Die Diskussion um die Verzerrungseffekte der Überhangmandate bezieht ihre besondere politische Brisanz aus den seit längerem zu beobachtenden Fragmentierungsprozessen des Parteiensystems. In dem Maße, in dem sich das zwischen den 1960er und 1980er Jahren sehr übersichtliche westdeutsche Parteiensystem mit zwei ähnlich starken Groß- und einer Kleinpartei zu einem immer weiter ausfransenden, gesamtdeutschen Parteiensystem mit einer wachsenden Zahl mandatsrelevanter parteipolitischer Akteure gewandelt hat, ist die Wahrscheinlichkeit von Überhangmandaten deutlich gewachsen. Hatten wir es dabei zunächst vornehmlich mit den Besonderheiten des ostdeutschen Parteiensystems zu tun, wo mit der Präsenz von drei großen politischen Akteuren die Wahrscheinlichkeit hoch war, dass eine Partei mit 30 bis 35 Prozent der Zweitstimmen dennoch nahezu sämtliche Wahlkreise in einem Bundesland gewinnen konnte, so ist inzwischen das Problem hinzugetreten, dass die beiden klassischen Großparteien - gemessen an den Bundestagswahlen 2009 nicht mehr unbedingt auf Augenhöhe konkurrieren. Wohl hat auch die Union gegenüber früheren Jahrzehnten deutliche Einbußen in der Wählergunst hinzunehmen; doch sind die Zustimmungsraten für die SPD erheblich stärker zurückgegangen. Sollte dieser Trend anhalten, wäre beim alten wie beim neuen Wahlrecht auch künftig von einer überproportionalen Vertretung der Union im Bundestag auszugehen - eine Überrepräsentanz, die bei einem entsprechend knappen Wahlausgang sogar entscheidenden Einfluss auf die Regierungsbildung haben könnte.

Natürlich wäre ein solcher Effekt des personalisierten Mehrheitswahlrechts nicht wünschenswert. Eine Stimmenminderheit, die gleichzeitig eine Mandatsmehrheit wäre, widerspräche einem verbreiteten Empfinden demokratischer Legitimation in Deutschland. Gleichwohl ist hier vor übertriebenen Befürchtungen zu warnen. Erstens ist nicht damit zu rechnen, dass ein zehnprozentiger Abstand zwischen stärkster und zweitstärkster Partei zur Regel wird; und zweitens sind mit solchen Eigenheiten des Wahlrechts auch Chancen zur Gegenwehr in Form von Bündnissen und Absprachen zwischen den Oppositionsparteien verbunden. Würden etwa Sozialdemokraten und Grüne bei Bundestagswahlen in vielen 
Wahlkreisen zu Absprachen über gemeinsame Kandidaten kommen, so würden wahrscheinlich am Ende eher sie von Überhangmandaten profitieren. Im Gegensatz zu vielen anderen demokratischen Systemen ist dem politischen System der Bundesrepublik eine solche Kultur von Wahlbündnissen und gemeinsamen Kandidaten bislang fremd. Aber wäre es denn so schrecklich, wenn das Wahlrecht unter den Bedingungen eines deutlich größten politischen Akteurs, der zugleich aber weit von der absoluten Mehrheit entfernt bleibt, zu solchen Veränderungen führte?

Dass es bei der Umrechnung von Stimmenergebnissen in Mandate zu nicht unerheblichen Verzerrungen kommt, ist im Übrigen keineswegs ungewöhnlich, wie ein Vergleich zeigt: Ganz selbstverständlich ist dies in Systemen mit Mehrheitswahlrecht. In Großbritannien hat 1997 ein Stimmenanteil von 43 Prozent ausgereicht, um der Labour Party einen Erdrutschwahlsieg mit fast zwei Dritteln der Mandate zu sichern. In Frankreich haben UMP und Gaullisten 2007 mit 45 Prozent der Stimmen knapp 60 Prozent der Mandate errungen. 2002 waren es bei nur 33 Prozent der Stimmen sogar noch mehr. Sozialisten und Grüne zusammen hatten damals eine ganz knappe Stimmenmehrheit, aber nicht einmal die Hälfte der Sitze von UMP und Gaullisten. In Italien ist 2005 ein Proportionalwahlsystem eingeführt worden, das die Partei oder das Bündnis mit relativer Mehrheit ausdrücklich begünstigt. In der Folge erreichte Romano Prodis Wahlbündnis „Unione Olivo“ 2006 mit 49,8 Prozent 340 Sitze in der Abgeordnetenkammer, Silvio Berlusconis „Casa della libertà “ mit 49,7 Prozent nur 277. 2008 reichten Berlusconi umgekehrt 46,8 Prozent zu der klaren absoluten Mehrheit von 344 der 630 Mandaten. Auch das spanische Verhältniswahlrecht bringt erhebliche Verzerrungseffekte. Es begünstigt die Provinzen mit relativ niedriger Bevölkerungszahl und hat mehrfach sowohl die Sozialisten als auch die Volkspartei mit einer absoluten Mehrheit der Mandate ausgestattet, obgleich ihre Stimmenanteile mit 44 beziehungsweise 45 Prozent deutlich darunter lagen und 99 beziehungsweise 96 Prozent der Stimmen für die Mandatsverteilung herangezogen wurden.

Aus einer internationalen Vergleichsperspektive erscheint die Aufgeregtheit, mit der die Diskussion über die Wahlrechtsreform hierzulande geführt wird, weit übertrieben. Anderswo hat man weniger Probleme, mit Mandatsmehrheiten für die stärkste Partei oder den stärksten Parteienblock umzugehen, die keine Stimmenmehrheiten erzielen.

\section{Demokratisches Wahlrecht in fragmentierten Parteiensystemen}

Das personalisierte Verhältniswahlrecht hat über viele Jahrzehnte die ihm zugedachten verschiedenen Funktionen geradezu ideal erfüllt. Es hat für eine annähernd exakte Widerspiegelung der gesellschaftlichen Grundströmungen in den Parlamenten ebenso gesorgt wie für die Rückbindung der politischen Akteure in regionale und lokale Strukturen. In dem Maße, in dem es trotz Sperrklausel die wachsende Ausdifferenzierung des Parteiensystems nicht aufhalten kann, zeigen sich nun seine Schwächen. Ein ideales Austarieren der verschiedenen Funktionen zwischen Legitimation und politischer Handlungsfähigkeit kann dieses Wahlrecht so wenig garantieren wie fast alle anderen.

Insoweit stellt sich natürlich die Frage nach der relativ besten Lösung für die Zukunft. Dabei spricht Einiges für moderate Korrekturen am bestehenden System. Der immer wieder einmal vernehmbare Ruf nach einem Mehrheitswahlrecht hat keine realistische Chance und entspricht auch nicht der in der Politischen Kultur hierzulande tief verwurzelten Vor- 
stellung von der vordringlichen Bedeutung proportional angemessener Umsetzung von Stimmen in Mandate. Ähnliches gilt für das Grabenwahlrecht, das zwar Überhangmandate unmöglich macht, weil der Mandatsanteil der Parteien durch die Zweitstimme nur auf die Hälfte der Parlamentssitze berechnet wird, aber im Ergebnis weit größere Verzerrungen zur Folge hätte als die Wahlrechtskonstruktion mit Überhangmandaten. Wäre 2009 in Deutschland nach dem Grabenwahlrecht gewählt worden, besäße die Union heute eine komfortable absolute Mehrheit.

Die relativ beste Variante für die Zukunft aber bestünde in der Schaffung von Zweierwahlkreisen und ihrer Verrechnung mit dem Zweitstimmenergebnis. Wenn künftig nicht ein, sondern zwei Direktkandidaten in Wahlkreisen gewählt würden, würde dies die Gefahr von Verzerrungseffekten durch Überhangmandate so gut wie ausschließen. Es erforderte allerdings eine erhebliche Verminderung der Wahlkreise, was in großen und bevölkerungsreichen Flächenstaaten Schwierigkeiten aufwirft. Wer aber die Verzerrungseffekte von Überhangmandaten beseitigen will, ohne die für die deutsche Wahlrechtstradition spezifische Mischung des Verhältniswahlrechts mit Elementen des Mehrheitswahlrechts aufzugeben, sollte sich solchen Modellen zuwenden. Wäre zum Beispiel 2009 statt in 299 Einerwahlkreisen in 150 Zweierwahlkreisen gewählt worden, wären keine Überhangmandate angefallen.

Erster Kandidat für den Gewinn von Zusatzmandaten wäre in einem solchen Wahlrecht die zweitstärkste Partei. Allerdings müsste dazu die SPD noch einmal deutlich unter die beim letzten Mal errungenen 23 Prozent fallen, ohne dass zugleich dritte Parteien Direktmandate in nennenswertem Umfang erreichten. Das aber erscheint extrem unwahrscheinlich. Hinzu kommt, dass eine derart weitreichende Zersplitterung des Parteiensystems, die einen solchen Fall entstehen ließe, Grundfragen von Stabilität und politischer Handlungsfähigkeit des demokratischen Systems insgesamt aufwerfen würde.

Geht man davon aus, dass die Fragmentierung des Parteiensystems weiter anhält, erscheint ein Wahlsystem mit Zweierwahlkreisen allen anderen überlegen, zumal es den Gesichtspunkten ausgewogener regionaler Mandatsverteilung Rechnung trüge und angesichts der derzeitigen politischen Kräfteverhältnisse neben Union und SPD auch Grünen und Linken realistische Chancen auf etliche Direktmandate geben würde.

Will man aber in den Bahnen des alten Wahlrechts bleiben und sich mit Korrekturen in den Details der Umrechnung begnügen, erscheint der Vorschlag der Regierungsfraktionen sehr kompliziert, aber keineswegs so abwegig und nur von Eigeninteressen diktiert, wie das von manchen derzeit behauptet wird. Die Vorschläge der Opposition sind jedenfalls keineswegs besser: Die Vergabe von Ausgleichsmandaten würde den Bundestag unnötig aufblähen und den Gesichtspunkten ausgewogener regionaler Verteilung der Mandate und regionaler Erfolgswertgleichheit der Stimmen noch weniger gerecht als das derzeitige System der Vergabe von Überhangmandaten ohne Ausgleich. Die Verrechnung von Überhang- und Listenmandaten in anderen Bundesländern widerspricht dem Grundsatz ausgewogener regionaler Mandatsverteilung ebenso, wie die Nichtvergabe von Direktmandaten ein systemwidriger Verstoß gegen den Grundsatz des gleichen Erfolgswertes jener Stimmen wäre, die in einem Wahlkreis für den Wahlkreissieger abgegeben worden sind. Gemessen daran erscheint der Vorschlag von Union und FDP weniger problematisch.

Eine befriedigende Lösung der Verzerrungsproblematik, die mit der Entstehung von Überhangmandaten regelmäßig verbunden ist, ist jedoch bei anhaltender Schwäche der Volksparteien eher möglich, wenn das System der Einerwahlkreise durch Zweierwahlkreise ersetzt würde. 